partially buried or rested on the surface. But particle-size is not all. Smooth-grained mixtures are sought after, sharp particles avoided; again, sands with silt or organic matter in excess of a certain amount are not favoured, but neither are those which lack micro-organisms on the surfaces of the grains. It is shown that the particular size-range of particle mixtures chosen by the lancelets are those with a high porosity, that is to say, they have a system of canal spaces within which water may circulate freely.
Thus, in the event, the lancelets select substrata by their texture, but benefit by other propertins dependent on texture, such as free drainage.

The community concept, in so far as it applies to animals living in the deposits of the ocean floor, has long been known to be founded upon the selectioa by animals of soils of varying texture and particle size. Few investigations in recent years have been more revealing of the bases of this selection than those described in the last of the five papers here reviewed.
J. E. Smitr

\title{
IMMUNOLOGICAL ASPECTS OF ALLERGIC DISORDERS
}

"I MMUNOLOGICAL Aspects of Allergic Disorders" was the subject of the symposium of the autumn meeting of the British Society for Immunology, held jointly with the British Association of Allergists, at the Welleome Building, London, on November 14.

A. W. Frankland introduced the subject as a clinician engaged in the treatment of 'allergic' disorders, for which antigen-antibody reactions are considered responsible. Seasonal pollen asthma, the least complicated allergic disease, responds equally well in 90 per cent of subjects to pre-seasonal treatment with a high dosage of an extract of the pollen of a single grass as with extracts of mixed grass, illustrating the need for studies of pollen antigens. A beneficial effect, however, is also found in between one-third and one-half of the patients treated with saline injections.

The significance of reactions to skin tests showing the presence of reaginic antibodies capable of sensitizing the skin requires clinical confirmation. In 50 per cent of subjects sensitive to pollen skin tests, observed over a period of ten years, clinical sensitivity does not appear to become important. Inhalation tests may confirm the presence of clinical sensitivity.

The clear description of allergic reactions is desir. able. Immediate reactions are seen in pollen sensitivity; delayed reactions are of the tuberculin type. An unexplained relationship between these is present in reactions to insect bites. The first bites are usually innocuous, but after a time they may excite reactions of the delayed type. At a later stage, reactions of the immediate type appear, and still later, in some cases, there may be a complete absence of reaction to the bites.

R. Augustin, in a paper entitled "Allergens and othor Pollen Antigens", reported her investigations into the relationship of grass pollen antigens, which excite in rabbits the production of precipitating antibodies, and those grass pollen antigens, which are allergenic for man, reacting with the reaginic but non-precipitating antibodies which sensitize the skin, as shown by reactions to skin tests. The complicated pattern of antigen - antibody precipitation lines produced in agar-gel diffusion tosts with rabbit antisera were studied for their relevance to allergenic activity in skin tests on man. Enzymic and other studies showed that the antigens of timothy and cocksfoot pollen that were most highly electrically charged were not significantly related to skin-test allergens. The main allergens were found in those antigens having the electrophoretic mobilities and salting out properties of human serum $\alpha$ - and $\beta$-globulins. These antigens, on one hand, were precipitated with rabbit antiserum and, on the other, accounted for almost all the allergenic activity in man. The concentrated fractions obtained in this way contained 10-100 million Noon units/mgm. ( $1 \mathrm{mgm}$. pollen equals 1,000 Noon units by definition). Coupled preparations of this active allergenic material with red cell stromata and hide powder (Baird and Tatlock) were effective in removing, specifically, the reaginic antibodies from the serum, as shown by passive transfer tests, thus providing a method for demonstrating the presence of reagins.

J. G. Feinberg and H. Grayson discussed the results of "A New Look at the Antibodies to so-called 'Common' Antigens of Grass Pollens". Using antibody gradients and eccentric patterns of precipitation, they showed that rabbit antibodies to pollens have a greater affinity for homospecific than for heterospecific pollens. A similar but greater homospecificity is shown with rabbit antisera to ovalbumin and ovalbumin tagged by means of arsenilic acid. It was suggested that pollen treatment should be based on homospecific pollen allergens.

Some observations on the antibodies related to ragweed pollen were discussed by $B$. Rose, who with Sehon and his colleagues have found that there are four antibodies in the serum of sensitive patients. Erythrocytes coated with antigen provided a highly sensitive and specific method for demonstrating nonprecipitating antibodies. Two of the antibodies found were skin-sensitizing, one was a blocking antibody, and one a hæmagglutinating antibody. The blocking antibody was found in the slow-moving $\gamma_{2}$-globulin and the skin-sensitizing antibody in the fast-moving $\gamma_{1}$-globulin. The skin-sensitizing antibody also moved with the fast-sedimenting $S 19$ fraction. The hæmagglutinating antibodies are considered to be similar to the ordinary divalent antibodies and were found in all serum fractions con. taining skin-sensitizing and/or blocking antibodies.

M. Loveless discussed the reproducibility and practical value of the conjunctival test for estimating the degree of hypersensitivity to ragweed pollen. Reactions to increasing concentrations were observed, and a good correlation was found between the degree of hypersensitivity and the titre of blocking antibody present in the serum after treatment by injection. The conjunctival test was used as a guide to the safe dosage of pollen for injection in a 'repository' form, in emulsion of liquid paraffin and 'Arlacel'. Such a guide is necessary since the dosage of pollen in the limited number of injections of repository required is far higher than the initial doses of aqueous extracts used for conventional methods of pollen treatment. 
Investigations into allergic hypersensitivity in man to Aspergillus fumigatus were described by $\mathbf{J}$. Pepys, R. W. Riddell, K. M. Citron, Y. M. Clayton and E. I. Short. Allergic reactions to the ubiquitous and perennial spores of $A$. fumigatus consist of rhinitis, conjunctivitis and asthma. The fungus also causes respiratory infections, associated with pulmonary eosinophilia, consisting of transient, recurrent pulmonary infiltrations, febrile episodes and eosinophilia of the sputum and blood. A. fumigatus is found with a significantly greater statistical frequency in the sputum of asthmatics without pulmonary eosinophilia than in the sputum of patients with other respiratory disorders.

Hypersensitivity as the cause of the pulmonary eosinophilia was investigated. Skin-prick tests with extracts of $A$. fumigatus, flavus, nidulans, niger and terreus gave positive reactions to almost all species in nearly every hypersensitive subject. Culturemedium filtrates, one month old, were the most potent allergens, followed by the cell sap of fresh mycelia, and then by conventional carbol saline extracts of dried mycelium.

Prick tests produced immediate weal-reactions, followed after 3-12 hr. in a number of cases by a 'late' reaction. This reaction was occasionally quite extensive and differed macroscopically and in its more rapid appearance and resolution from the delayed tuberculin type of reaction. Histologically it showed early, intense and persistent infiltration of eosinophile cells, as compared with the perivascular infiltration of round cells in the tuberculin reaction. These features and the demonstration of precipitating antibodies in the serum of pationts by means of concentrated extracts of Aspergillus, especially $A$. fumigatus, suggest that the 'late' reaction is of the Arthus type.

Bronchial reactions to inhalation tests with extracts of Aspergillus were most evident in patients with pulmonary eosinophilia. Proof that the fungus plays a part in the clinical picture must rest, how. ever, not only on evidence of its presence in the sputum, but also on immunological confirmation.
Preliminary investigations into the immunochemistry of Cladosporium were reported by $\mathrm{J}$. Longbottom, B. Hayward and R. Augustin. Extracts of spores and mycelium were found to be equal in potency in skin tests. Their active components were found, as with pollen, to be nondialysable and to be associated with the protein-like material which did not move from the base-line in paper chromatography.

At least eight antigens were demonstrable in agar-gel tests with rabbit antisera to $C$. fulvum and $C$. herbarum. Antiserum to $C$. herbarum gave precipitation lines with the antigens of $C$. fulvum, but not vice versa. The Cladosporium antigens have the mobilities of serum albumins and $\alpha$ - and $\beta$-globulins. Of four antigens shown by immunoelectrophoresis in $C$. fulvum, three were found to be non-allergenic in man. Patients tested with the extracts and with proteolytic digests of the two species showed individual variations, as might be expected.

J. F. Ackroyd discussed a patient with allergic thrombocytopenic purpura due to antazoline. Antazoline added to the serum caused lysis of the platelets and the formation of a precipitate, both mediated by the same antibody. Taking the drug on two later occasions resulted in a rise in the fallen antibody titre. Formation of a precipitate at these times was not, as it had been previously, accompanied by complement fixation; and no cause could be found for this.

The observation that the formation of a precipitate with complement fixation was produced by adding the drug to serum, collected while the patient was taking sufficient antazoline to give high enough blood-levels to excite thrombocytopenia, suggests that the union of the drug and the platelets is very labile. This dissociability may explain why so fow persons develop purpura on taking antazoline and other drugs such as sedormid, quinine and quinidine, for which a similar lability has been observed.

J. Pepys

\title{
THE NEW FERODO RESEARCH LABORATORIES
}

\author{
BY DR R. C. PARKER
}

Research Director

$\mathrm{O}^{\mathrm{N}}$ November 21, some sixty-one years after $\mathrm{Mr}$. Herbert Frood, the founder of Ferodo Ltd., produced his first friction material, H.R.H. the Duke of Edinburgh formally opened the new Research Laboratories of the firm by unveiling a suitably inscribed bronze plaque. Work on current problems was afterwards inspected. In the following week a series of open days were arranged, one of which was reserved for the teaching staffs of universities, Iocal technical colleges and schools and for the staffs of research institutes.

The building (Fig. 1) is in the form of a splayed $E$ having a total floor area of $75,000 \mathrm{sq}$. $\mathrm{ft}$. The upper two stories of the front of the building house the Administration Department, a Plant Development Department and offices for senior managers, while the two-storied centre wing with a floor area of
12,000 sq. ft. houses the Physical, Chemical and Metallurgical Laboratories. All the internal partitions in both laboratories and offices are built on a five-foot module and can be readily mounted or removed as desired. The left-hand wing, covering 21,000 sq. ft., comprises an Engineering Test House, a workshop and a test car garage, and the right-hand wing, with an area of 18,000 sq. ft., contains pilot plant. The site of the building is such that when further space is required the length of the three projecting wings can be increased by 50 per cent. The building is pleasantly situated on the main road from Chapelen-le-Frith to Hayfield, and is set among landscape gardens.

The main heating of the offices and the laboratories is by warm water that circulates through special steel coils embedded in the ceilings. Supplementirg 